\title{
Consensus Statement on the Adoption of the COPE Guidelines
}

We, the undersigned Editors of the member journals of the Surgery Journal Editors Group (SJEG), in the furtherance of integrity in surgical and scientific publication, agree to adopt the guidelines established by the Committee on Publication Ethics (COPE). ${ }^{1}$ The COPE guidelines represent a means of addressing a variety of ethical concerns, including duplicate publication and authorship misconduct issues, which have, unfortunately, become more prevalent.

This statement is being simultaneously published in the respective journals of the members of the Surgery Journal Editors Group, as follows:

American Journal of Surgery Kirby I. Bland, MD.

Annals of Surgery Layton F. Rikkers, MD, Keith D. Lillemoe, MD.

Annals of Surgical Oncology Charles M. Balch. MD, Mark S. Roh, MD.

Annals of Thoracic Surgery L. Henry Edmunds, Jr., MD. Archives of Surgery Julie Freischlag, MD.

BJS Derek Alderson, MD, Jonothan J. Earnshaw, MD. Burns Steven E. Wolf, MD.

Canadian Journal of Surgery Edward J Harvey, MD, Garth L. Warnock, MD.

Der Chirurg J. R. Siewert, MD.

Digestive Surgery Markus W. Büchler, MD, John P. Neoptolemos, MD.

Diseases of the Colon and Rectum Robert D. Madoff, MD.

ePlasty Stephen M. Milner MD.

Female Pelvic Medicine \&.

Reconstructive Surgery Alfred E. Bent, MD.

$H P B$ O. James Garden, MD.

$H P B$ Surgery Robin C. Williamson, MD.

Journal of the American College of Surgeons Timothy J. Eberlein, MD.

Journal of Burn Care and Research Richard Gamelli, MD.

Journal of Gastrointestinal Surgery John L. Cameron, MD, Jeffrey B. Matthews, M.D., Charles J. Yeo, M.D.
Journal of Hepato-Biliary-Pancreatic Sciences Tadahiro Takada, MD.

Journal of Laparoendoscopic \& C. Daniel Smith, MD. Advanced Surgical Techniques.

Journal of Pediatric Surgery Jay L. Grosfeld, MD.

Journal of Plastic \& Reconstructive Surgery Rod J. Rohrich, MD.

Journal of Surgical Education John A. Weigelt, MD. Journal of Surgical Research David McFadden, MD, Wiley W. Souba, MD.

Journal of Thoracic \& Cardiovascular Surgery Lawrence H. Cohn, MD.

Journal of Trauma Basil A. Pruitt, Jr, MD.

Journal of Vascular Surgery Anton N. Sidawy, MD, MPH, Bruce A. Perler, MD, MBA.

Nutrition Michael M. Meguid, MD, PhD.

Pediatric Surgery International Arnold G. Coran, MD, Prem Puri, MD.

Surgery Andrew L. Warshaw, MD, Michael Sarr, MD. Surgery for Obesity \& Related Diseases Harvey J. Sugerman, MD.

Surgical Endoscopy Alfred Cuschieri, MD, Mark Talamini, MD.

Surgical Innovation Adrian Park, MD, Lee Swanstrom, MD.

Surgical Laparoscopy, Endoscopy \& Percutaneous Techniques Maurice E. Arregui, MD, Carol ScottConner, MD. PhD.

The American Surgeon J. David Richardson, MD.

World Journal of Surgery John G. Hunter, MD.

Zentralblatt für Chirurgie Hans Lippert, MD, Ulrich Hopt, MD, Karl-Walter Jauch, MD.

(c) Society of Surgical Oncology 2010

\section{REFERENCE}

1. COPE Committee on Publication Ethics. http://publicationethics. org/guidelines. 